\title{
TRANSIENT BEHAVIOR OF SINGLE-SERVER QUEUEING PROCESSES WITH ERIANG INPUT
}

\author{
BY \\ LAJOS TAKÁCS(')
}

1. Introduction. We are concerned with single server queueing processes. Let us suppose that customers arrive at a counter at the instants $\tau_{1}^{*}, \tau_{2}^{*}, \cdots$, $\tau_{n}^{*}, \cdots$ where the inter-arrival times $\tau_{n+1}^{*}-\tau_{n}^{*}\left(n=0,1,2, \cdots, \tau_{0}^{*}=0\right)$ are identically distributed, independent random variables with distribution function

$$
F_{m}(x)= \begin{cases}1-\sum_{j=0}^{m-1} e^{-\lambda x} \frac{(\lambda x)^{j}}{j !} & \text { if } x \geqq 0, \\ 0 & \text { if } x<0 .\end{cases}
$$

We say that $\left\{\tau_{n}^{*}\right\}$ is an Erlang process. The customers will be served by a single server in the order of their arrival. Suppose that the server is idle if and only if there is no customer waiting at the counter. Denote by $\chi_{n}^{*}$ the service time of the $n$th customer $(n=1,2, \cdots)$. It is supposed that $\left\{\chi_{n}^{*}\right\}$ is a sequence of identically distributed, independent, positive random variables with distribution function

$$
P\left\{\chi_{n}^{*} \leqq x\right\}=H(x)
$$

and that $\left\{\chi_{n}^{*}\right\}$ is independent of $\left\{\tau_{n}^{*}\right\}$.

Denote by $\eta^{*}(t)$ the virtual waiting time at the instant $t$, i.e., $\eta^{*}(t)$ is the time which a customer would wait if he joined the queue at the instant $t$. $\eta^{*}(0)$ is the initial occupation time of the server. Define $\eta_{n}^{*}=\eta\left(\tau_{n}^{*}-0\right)$, i.e., $\eta_{n}^{*}$ is the waiting time of the $n$th customer.

Denote by $\xi^{*}(t)$ the queue size at the instant $t$, i.e., $\xi^{*}(t)$ is the number of customers waiting or being served at the instant $t . \xi^{*}(0)$ is the initial queue size. Further let us denote by $\tau_{1}^{\prime}, \tau_{2}^{\prime}, \cdots, \tau_{n}^{\prime}, \cdots$ the instants of the successive departures and define $\xi_{n}^{*}=\xi^{*}\left(\tau_{n}^{\prime}+0\right),(n=1,2, \cdots)$, i.e., $\xi_{n}^{*}$ is the queue size immediately after the $n$th departure. If there is a departure at $t=0$ then we write $\tau_{0}^{\prime}=0$ and $\xi_{0}^{*}=\xi^{*}(+0)$.

Finally, denote by $G_{n}^{*}(x)$ the probability that a busy period consists of $n$ services and its length is at most $x$.

We are interested in the investigation of the stochastic behavior of the waiting time, the queue size, and the busy period of this process. We shall see,

Received by the editors April 27, 1960 and, in revised form, June 15, 1960.

(1) This research was sponsored in part by the Office of Naval Research under Contract Number Nonr-266(33), Project Number NR 042-034. Reproduction in whole or part is permitted for any purpose of the United States Government. 
however, that if we know the stochastic behavior of the process defined below, then that of the above process can be deduced immediately. The second process is defined in such a way that it has a wider state space than the first one, i.e., the first process is imbedded in the second one. It is an advantage that the second process has more Markovian properties than the first one.

To define the second process let us suppose that customers arrive at a counter at the instants $\tau_{1}, \tau_{2}, \cdots, \tau_{n}, \cdots$ where the inter-arrival times $\tau_{n+1}-\tau_{n}\left(n=1,2, \cdots ; \tau_{0}=0\right)$ are identically distributed, independent random variables with distribution function

$$
F(x)=\left\{\begin{array}{cl}
1-e^{-\lambda x} & \text { if } x \geqq 0, \\
0 & \text { if } x<0,
\end{array}\right.
$$

i.e., $\left\{\tau_{n}\right\}$ is a Poisson process. The customers will be served by a single server in batches of size $m$ in the order of their arrival. The server is idle if and only if fewer than $m$ customers are present. Denote by $\chi_{n}$ the service time of the $n$th batch $(n=1,2, \cdots)$. The service times $\chi_{n}(n=1,2, \cdots)$ are identically distributed, independent, positive random variables with distribution function

$$
P\left\{\chi_{n} \leqq x\right\}=H(x)
$$

and independent of $\left\{\tau_{n}\right\}$.

Denote by $\eta(t)$ the occupation time of the server at the instant $t$, i.e., $\eta(t)$ is the time which elapses from $t$ until the server becomes idle for the first time if no customers join the queue after the time $t . \eta(0)$ is the initial occupation time of the server.

Denote by $\xi(t)$ the queue size at the instant $t$, i.e., $\xi(t)$ is the number of customers waiting or being served at the instant $t . \xi(0)$ is the initial queue size. We say that the system is in state $E_{k}$ at the instant $t$ if $\xi(t)=k$.

Denote by $\tau_{1}^{\prime}, \tau_{2}^{\prime}, \cdots, \tau_{n}^{\prime}, \cdots$ the instants of the successive departures and define $\xi_{n}=\xi\left(\tau_{n}^{\prime}+0\right)(n=1,2, \cdots)$. If there is a departure at $t=0$ then we write $\tau_{0}^{\prime}=0$ and $\xi_{0}=\xi(+0)$.

Denote by $\eta_{n}(n=1,2, \cdots)$ the waiting time of the customer arriving last among those who are served in the $n$th batch. (We note that if $\eta_{n}$ denotes the waiting time of the $r$ th arriving customer among those who are served in the $n$th batch, then the sequence $\left\{\eta_{n}\right\}$ follows a similar stochastic law as the former one, only the initial distribution of $\eta_{1}$ is changed.)

Finally, denote by $G_{n}(x)$ the probability that the busy period consists of $n$ services and its length is at most $x$.

If we identify every $m$ th arrival in the second process with an arrival in the first process, i.e., we suppose that $\tau_{n}^{*}=\tau_{n m}$ and similarly if we identify the service time of the $n$th batch in the second process with the service time of the $n$th customer in the first process i.e., $\chi_{n}^{*}=\chi_{n}(n=1,2, \cdots)$ and further we suppose that the initial states are also in agreement, then the second 
process is reduced to the first one. For, $F_{m}(x)$ is the $m$ th iterated convolution of $F(x)$ with itself.

Comparing the two processes we see that the waiting time and the busy period follow the same probability laws in both processes, namely $\eta^{*}(t)=\eta(t)$, $\eta_{n}^{*}=\eta_{n}$ whenever $\xi(0)=0(\bmod m)$ and $G_{n}^{*}(x)=G_{n}(x)$. Further, the departures also agree. However the queue sizes are different, namely

$$
\xi^{*}(t)=\left[\frac{\xi(t)}{m}\right] \text { and } \xi_{n}^{*}=\left[\frac{\xi_{n}}{m}\right]
$$

where $[a]$ means the greatest integer $\leqq a$.

In the following we shall consider only the second process and determine the stochastic behavior of the waiting time, the queue size and that of the busy period.

The transient behavior of the process $\left\{\eta_{n}\right\}$ can be deduced from more general theorems proved by F. Pollaczek $[6 ; 7]$. The stochastic law of the busy period has been given by B. W. Conolly [2] and for a more general case by F. Pollaczek [8]. The asymptotic behavior of the waiting time and that of the queue size has been treated by F. Pollaczek [6;7], A. J. Fabens [3], and in a special case by R. R. P. Jackson and D. G. Nickols [4].

Notation. Denote by $M_{j}(t)(j=0,1,2, \cdots)$ the expectation of the number of transitions $E_{j} \rightarrow E_{j+1}$ occurring in the time interval $(0, t]$ and denote by $N_{j}(t)(j=0,1,2, \cdots)$ the expectation of the number of transitions $E_{j+m} \rightarrow E_{j}$ occurring in the time interval $(0, t]$.

The Laplace-Stieltjes transform of the distribution function of the service time is denoted by

$$
\psi(s)=\int_{0}^{\infty} e^{-s x} d H(x)
$$

which is convergent if $\Re(s) \geqq 0$. The average service time is

$$
\alpha=\int_{0}^{\infty} x d H(x)
$$

The distribution function of the occupation time is denoted by

$$
W(t, x)=P\{\eta(t) \leqq x\}
$$

and its Laplace-Stieltjes transform, by

$$
\Omega(t, s)=E\left\{e^{-8 \eta(t)}\right\}=\int_{0}^{\infty} e^{-s x} d_{x} W(t, x)
$$

for $\Re(s) \geqq 0$. The probability distribution of the queue size is denoted by 


$$
P_{j}(t)=P\{\xi(t)=j\} \quad(j=0,1,2, \cdots)
$$

and its Laplace transform, by

$$
\Pi_{j}(s)=\int_{0}^{\infty} e^{-s t} P_{j}(t) d t
$$

for $\Re(s)>0$.

2. An auxiliary theorem. Throughout this paper we need

LEMmA 1. If (a) $\Re(s) \geqq 0,|w|<1$ or (b) $\Re(s)>0,|w| \leqq 1$ or (c) $\lambda \alpha>m$ and $\Re(s) \geqq 0,|w| \leqq 1$ then the equation

$$
z^{m}=\operatorname{rr}(s+\lambda(1-z))
$$

has exactly $m$ roots $z=\gamma_{r}(s, w)(r=1,2, \cdots, m)$ in the unit circle $|z|<1$. We have

$$
\gamma_{r}(s, w)=\sum_{j=1}^{\infty} \frac{(-\lambda)^{j-1}\left(\epsilon_{r} w^{1 / m}\right)^{j}}{j !}\left(\frac{d^{j-1}[\psi(\lambda+s)]^{j / m}}{d s^{j-1}}\right)
$$

where $\epsilon_{r}=\exp (2 \pi i r / m)(r=1,2, \cdots, m)$ are the mth roots of unity.

If $\gamma_{r}(s, w)$ is defined by (4) for $\Re(s) \geqq 0$ and $|w| \leqq 1$, then in this domain $\gamma_{r}(s, w)$ is a regular function of $s$ and $w,\left|\gamma_{r}(s, w)\right| \leqq 1$ and $z=\gamma_{r}(s, w)$ satisfies the equation

$$
z=\epsilon_{r}[\operatorname{wr} \psi(s+\lambda(1-z))]^{1 / m} .
$$

The roots $\gamma_{r}(s, w)(r=1,2, \cdots, m)$ are distinct if $w \neq 0$.

Proof. In cases (a) and (b) we have $|\boldsymbol{w} \psi(s+\lambda(1-z))|<(1-\epsilon)^{m}$ if $|z|=1-\epsilon$ and $\epsilon$ is a sufficiently small positive number. Consequently by Rouchés theorem (3) has exactly $m$ roots in the circle $|z|<1-\epsilon$. In case (c) we have $\psi(\lambda \epsilon)<(1-\epsilon)^{m}$ if $\epsilon$ is a sufficiently small positive number. For, if $0 \leqq \epsilon \leqq 1$ then $\psi(\lambda \epsilon)$ and $(1-\epsilon)^{m}$ are monotone decreasing functions of $\epsilon$, they agree at $\epsilon=0$, and their derivatives at $\epsilon=0$ are $-\lambda \alpha$ and $-m$ respectively. Hence $|\boldsymbol{w} \psi(s+\lambda(1-z))| \leqq \psi(\lambda \epsilon)<(1-\epsilon)^{m}$ if $|z|=1-\epsilon$ and $\epsilon$ is small enough, and by Rouché's theorem (3) has exactly $m$ roots in the circle $|z|<1-\epsilon$. Forming the Lagrange expansion of $z$ by (5) we obtain (4). (Cf. e.g. E. T. Whittaker and G. N. Watson $\left[9\right.$, p. 132].) Accordingly, each $\gamma_{r}(s, w)(r=1,2, \cdots, m)$ is the only root in $z$ of the equation (5) in the unit circle. Note that the roots $\gamma_{r}(s, w)(r=1,2, \cdots, m)$ are regular functions of $s$ and $w$ and by analytical continuation they can be defined also in the case $\lambda \alpha \leqq m$ for $\Re(s) \geqq 0$ and $|w| \leqq 1$ without changing (4). We have always $\left|\gamma_{r}(s, w)\right| \leqq 1(r=1,2, \cdots, m)$ if $\Re(s) \geqq 0$ and $|w| \leqq 1$. The equation (3) has at most one root (possibly multiple) on the unit circle $|z|=1$, namely $z=1$ is a root if $\operatorname{wr}(s)=1$. Note also that $\gamma_{r}(s, w)=0$ if and only if $w=0$. If $w \neq 0$ then the roots $\gamma_{r}(s, w)$ $(r=1,2, \cdots, m)$ are distinct. This completes the proof. 
Let us introduce the following abbreviations. Let $\gamma_{r}(s)=\gamma_{r}(s, 1), g_{r}(w)$ $=\gamma_{r}(0, w)$ and $\omega_{r}=\gamma_{r}(0,1)(r=1,2, \cdots, m)$. They satisfy the equations $z^{m}=\psi(s+\lambda(1-z)), z^{m}=u \psi(\lambda(1-z))$ and $z^{m}=\psi(\lambda(1-z))$ respectively.

Finally, we remark that by forming the Lagrange expansion of $\left[\gamma_{r}(s, w)\right]^{n}(r=1,2, \cdots, m)$ we can prove the following formula

$$
\sum_{r=1}^{m}\left[\gamma_{r}(s, w)\right]^{n}=n \sum_{j \geqq n / m} \frac{w^{i} \lambda^{m j-n}}{j(m j-n) !} \int_{0}^{\infty} e^{-(\lambda+s) x} x^{m j-n} d H_{j}(x)
$$

where $H_{j}(x)$ denotes the $j$ th iterated convolution of $H(x)$ with itself. By using this formula we can obtain explicit formulas for the probabilities considered in this paper.

Lemma 2. Let $\omega_{r}=\gamma_{r}(0,1)$ where $\gamma_{r}(s, w)$ is defined by (4). If $\lambda \alpha>m$ then $\omega_{1}, \omega_{2}, \cdots, \omega_{m}$ are the $m$ roots in $z$ of the equation

$$
z^{m}=\psi(\lambda(1-z))
$$

in the unit circle $|z|<1$. If $\lambda \alpha \leqq m$ then $\omega_{1}, \omega_{2}, \cdots, \omega_{m-1}$ are the $m-1$ roots in $z$ of $(7)$ in the unit circle $|z|<1$ while $\omega_{m}=1$.

Proof. If $\lambda \alpha>m$ then by Lemma 1 we have $\left|\omega_{r}\right|<1(r=1,2, \cdots, m)$. If $\lambda \alpha \leqq m$ then it follows from (5) that $\left|\omega_{r}\right|<1$ if $r=1,2, \cdots, m-1$ and a probabilistic argument shows that $\omega_{m}=1$ (cf. Remark 4 and Remark 5).

If $\omega_{m}=1$ then using (3) we get

$$
\gamma_{m}^{\prime}(0)=\left\{\begin{array}{cl}
\alpha /(\lambda \alpha-m) & \text { if } \lambda \alpha<m \\
\infty & \text { if } \lambda \alpha=m
\end{array}\right.
$$

and

$$
g_{m}^{\prime}(1)=\left\{\begin{array}{cl}
1 /(m-\lambda \alpha) & \text { if } \lambda \alpha<m \\
\infty & \text { if } \lambda \alpha=m
\end{array}\right.
$$

REMARK 1. The functions

$$
\zeta_{r}(s, w)=s+\lambda\left[1-\gamma_{r}(s, w)\right] \quad(r=1,2, \cdots, m)
$$

satisfy in $\zeta$ the equation

$$
\left(\frac{\lambda+s-\zeta}{\lambda}\right)^{m}=w \psi(\zeta)
$$

if $\Re(s) \geqq 0$ and $|w| \leqq 1$. Conversely in cases (a), (b), and (c) it follows by Rouché's theorem that (11) has exactly $m$ roots in the domain $\Re(\zeta)>0$ or

$$
\zeta=(\lambda+s)-\epsilon_{r} \lambda[w \psi(\zeta)]^{1 / m} \quad(r=1,2, \cdots, m)
$$

has exactly one root $\zeta=\zeta_{r}(s, w)$ in the domain $\Re(\zeta)>0$. For, 


$$
|\operatorname{ve}(\zeta)|<\left|\left(\frac{\lambda+s-\zeta}{\lambda}\right)^{m}\right|
$$

if $\Re(\zeta)=\epsilon>0$ and $\epsilon$ is small enough or $\Re(\zeta) \geqq \epsilon$ where $\epsilon$ is a sufficiently small positive number and $|\zeta|$ is large enough.

Let us write $\zeta_{r}(s)=\zeta_{r}(s, 1)$. Clearly $\zeta_{r}(0,1)=\zeta_{r}(0)=\lambda\left(1-\omega_{r}\right)$.

3. The transient behavior of the process $\{\eta(t)\}$. The process $\{\eta(t)\}$ can be described as follows. $\eta(0)$ is the initial occupation time of the server and $\eta(t)$ decreases linearly with slope -1 until it jumps or reaches 0 . The jumps occur at the arrivals of every $m$ th customer and their magnitude is the service time of the corresponding batch. If $\eta(t)$ reaches 0 then it remains 0 until the queue size increases to $m$.

Let us define the random variables $\nu(t)(0 \leqq t<\infty)$ as follows: $\nu(t)$ assumes only the values $1,2, \cdots, m$ and $\xi(t) \equiv \nu(t)-1(\bmod m)$. The reason for introducing $\nu(t)$ lies in the fact that $\{\eta(t)\}$ in itself is not a Markov process, but the vector process $\{\eta(t), \nu(t)\}$ is Markovian, for which we can apply standard methods.

Let us introduce the following notation:

$$
W_{j}(t, x)=P\{\eta(t) \leqq x, \nu(t)=j\} \quad(j=1,2, \cdots, m)
$$

and

$$
\Omega_{j}(t, s)=\int_{0}^{\infty} e^{-8 x} d_{x} W_{j}(t, x) \quad(j=1,2, \cdots, m)
$$

Then we have

$$
W(t, x)=\sum_{j=1}^{m} W_{j}(t, x)
$$

and

$$
\Omega(t, s)=\sum_{j=1}^{m} \Omega_{j}(t, s) .
$$

Write $\hat{\Omega}_{j}(s)=\Omega_{j}(0, s)$ and $\hat{\Omega}(s)=\Omega(0, s)$ and introduce the generating function

$$
R(s, z)=\sum_{j=1}^{m} \hat{\Omega}_{j}(s) z^{j-1}
$$

which is determined by the initial condition.

Obviously

$$
W_{j}(t, 0)=P_{j-1}(t) \quad(j=1,2, \cdots, m)
$$


because $P\{\eta(t)=0, \nu(t)=j\}=P\{\xi(t)=j-1\}$ if $j=1,2, \cdots, m$.

Finally, for $|z| \leqq 1$ let us introduce the generating functions

$$
P(t, z)=\sum_{j=0}^{m-1} P_{j}(t) z^{j}
$$

and

$$
\Pi(s, z)=\sum_{j=0}^{m-1} \Pi_{j}(s) z^{j}
$$

We shall prove

THEOREM 1. The Laplace-Stieltjes transform of the distribution function of the occupation time,

$$
\Omega(t, \zeta)=E\left\{e^{-\zeta \eta(t)}\right\}=\int_{0}^{\infty} e^{-\zeta x} d_{x} W(t, x)
$$

is given by

$$
\begin{aligned}
\Omega(t, \zeta)= & \frac{[1-\psi(\zeta)]}{m \psi(\zeta)} \sum_{j=1}^{m} \frac{\epsilon_{j}[\psi(\zeta)]^{1 / m}}{1-\epsilon_{j}[\psi(\zeta)]^{1 / m}} \\
& \cdot\left[e^{s_{j} t} R\left(\zeta, \epsilon_{j}[\psi(\zeta)]^{1 / m}\right)-\zeta \int_{0}^{t} e^{s_{j}(t-u)} P\left(u, \epsilon_{j}[\psi(\zeta)]^{1 / m}\right) d u\right]
\end{aligned}
$$

where

$$
s_{j}=\zeta-\lambda+\lambda \epsilon_{j}[\psi(\zeta)]^{1 / m}
$$

and $\epsilon_{j}=\exp (2 \pi i j / m)(j=1,2, \cdots, m)$. If $\Re(s)>0$ and $|z| \leqq 1$ then the Laplace transform of $P(t, z)$ is

$$
\begin{aligned}
\Pi(s, z) & =\int_{0}^{\infty} e^{-s t} P(t, z) d t \\
& =\sum_{j=1}^{m} \frac{R\left(s+\lambda\left[1-\gamma_{j}(s)\right], \gamma_{j}(s)\right)}{s+\lambda\left[1-\gamma_{j}(s)\right]} \prod_{r \neq j}\left(\frac{z-\gamma_{r}(s)}{\gamma_{j}(s)-\gamma_{r}(s)}\right)
\end{aligned}
$$

where $z=\gamma_{r}(s)(r=1,2, \cdots, m)$ are the $m$ roots in $z$ of the equation

$$
z^{m}=\psi(s+\lambda(1-z))
$$

in the unit circle $|z|<1$.

Proof. A simple argument shows that

$$
W_{1}(t+\Delta t, x)=(1-\lambda \Delta t) W_{1}(t, x+\Delta t)+\lambda \Delta t \int_{0}^{x} H(x-y) d_{y} W_{m}(t, y)+o(\Delta t)
$$


and for $j=2,3, \cdots, m$

$$
W_{j}(t+\Delta t, x)=(1-\lambda \Delta t) W_{j}(t, x+\Delta t)+\lambda \Delta t W_{j-1}(t, x)+o(\Delta t),
$$

whence

$$
\frac{\partial W_{1}(t, x)}{\partial t}=\frac{\partial W_{1}(t, x)}{\partial x}-\lambda W_{1}(t, x)+\lambda \int_{0}^{x} H(x-y) d_{\nu} W_{m}(t, y)
$$

and for $j=2,3, \cdots, m$

$$
\frac{\partial W_{j}(t, x)}{\partial t}=\frac{\partial W_{j}(t, x)}{\partial x}-\lambda W_{j}(t, x)+\lambda W_{j-1}(t, x) .
$$

These equations hold for almost every $t$ and $x$. Forming the Laplace-Stieltjes transforms of (23) and (24) we get for $\Re(\zeta)>0$ that

$$
\frac{\partial \Omega_{1}(t, \zeta)}{\partial t}=(\zeta-\lambda) \Omega_{1}(t, \zeta)+\lambda \psi(\zeta) \Omega_{m}(t, \zeta)-\zeta P_{0}(t)
$$

and for $j=2,3, \cdots, m$

$$
\frac{\partial \Omega_{j}(t, \zeta)}{\partial t}=(\zeta-\lambda) \Omega_{j}(t, \zeta)+\lambda \Omega_{j-1}(t, \zeta)-\zeta P_{j-1}(t)
$$

Now let us introduce the following matrix notation:

$$
\boldsymbol{A}=\left\|\begin{array}{cccc}
(\zeta-\lambda) & 0 & \cdots & \lambda \psi(\zeta) \\
\lambda & (\zeta-\lambda) & \cdots & 0 \\
\cdot & \cdot & \cdots & 0 \\
0 & 0 & \cdots & \cdot \\
(\zeta-\lambda)
\end{array}\right\|
$$

and

$$
\boldsymbol{\Omega}(t, \zeta)=\left\|\begin{array}{c}
\Omega_{1}(t, \zeta) \\
\Omega_{2}(t, \zeta) \\
\vdots \\
\Omega_{m}(t, \zeta)
\end{array}\right\|, \quad \boldsymbol{P}(t)=\left\|\begin{array}{c}
P_{0}(t) \\
P_{1}(t) \\
\vdots \\
P_{m-1}(t)
\end{array}\right\|
$$

Combining (25) and (26) we get the following matrix differential equation

$$
\frac{\partial \boldsymbol{\Omega}(t, \zeta)}{\partial t}=\boldsymbol{A} \boldsymbol{\Omega}(t, \zeta)-\zeta \boldsymbol{P}(t)
$$

the solution of which is

$$
\mathbf{\Omega}(t, \zeta)=e^{A^{t}} \hat{\mathbf{\Omega}}(\zeta)-\zeta \int_{0}^{t} e^{A(t-u)} P(u) d u,
$$


where

$$
\hat{\mathbf{\Omega}}(\zeta)=\mathbf{\Omega}(0, \zeta)
$$

is determined by the initial condition and $\boldsymbol{P}(t)$ is yet to be determined. $\boldsymbol{P}(t)$ can be obtained by the requirement that

$$
\int_{0}^{\infty} e^{-s t} \mathbf{\Omega}(t, \zeta) d t
$$

is a regular vector function of $\zeta$ if $\Re(\zeta) \geqq 0$ and $\Re(s)>0$. Forming the Laplace transform of (29) we obtain

$$
\int_{0}^{\infty} e^{-s t} \mathbf{\Omega}(t, \zeta) d t=[A-s I]^{-1}\left[\hat{\mathbf{\Omega}}(\zeta)-\zeta \int_{0}^{\infty} e^{-s t} P(t) d t\right],
$$

where $I$ is the $(m, m)$ unit matrix. To determine the components of (30) express $\boldsymbol{A}$ in canonical form. The characteristic equation of $\boldsymbol{A}$ is

$$
|\boldsymbol{A}-s \boldsymbol{I}|=(\zeta-\lambda-s)^{m}-(-1)^{m} \lambda^{m} \psi(\zeta)=0
$$

and hence the proper values of $\boldsymbol{A}$ are

$$
s_{j}=s_{j}(\zeta)=\zeta-\lambda+\lambda \epsilon_{j}[\psi(\zeta)]^{1 / m} \quad(j=1,2, \cdots, m)
$$

where $\epsilon_{j}=\exp (2 \pi i j / m)(j=1,2, \cdots, m)$ are the $m$ th roots of unity. A simple calculation shows that

$$
\boldsymbol{A}=\left\|\alpha_{i j}\right\| \mathbf{\Lambda}\left\|\beta_{j k}\right\|
$$

where $\boldsymbol{\Lambda}$ is the diagonal matrix $\left[s_{1}, s_{2}, \cdots, s_{m}\right]$ and $\left\|\alpha_{i j}\right\|$ and $\left\|\beta_{j k}\right\|$ are inverse matrices for which

$$
\alpha_{i j}=\left(\frac{\lambda}{\lambda+s_{j}-\zeta}\right)^{i}
$$

and

$$
\beta_{j k}=\frac{1}{m}\left(\frac{\lambda+s_{j}-\zeta}{\lambda}\right)^{k}
$$

Thus

$$
[A-s I]^{-1}=\left\|\alpha_{i j}\right\|[\mathbf{\Lambda}-s I]^{-1}\left\|\beta_{j k}\right\|=\left\|\gamma_{i k}\right\|
$$

where

$$
\gamma_{i k}=\sum_{j=1}^{m} \frac{\alpha_{i j} \beta_{j k}}{s_{j}-s}=\frac{1}{m} \sum_{j=1}^{m}\left(\frac{\lambda+s_{j}-\zeta}{\lambda}\right)^{k-i} \frac{1}{\left(s_{j}-s\right)} .
$$

Finally by (30) we get 


$$
\begin{aligned}
& \int_{0}^{\infty} e^{-s t} \Omega_{i}(t, \zeta) d t=\sum_{k=1}^{m} \gamma_{i k}\left[\hat{\Omega}_{k}(\zeta)-\zeta \Pi_{k-1}(s)\right] \\
& =\frac{1}{m} \sum_{k=1}^{m} \sum_{j=1}^{m}\left(\frac{\lambda+s_{j}-\zeta}{\lambda}\right)^{k-i} \frac{\left[\hat{\Omega}_{k}(\zeta)-\zeta \Pi_{k-1}(s)\right]}{\left(s_{j}-s\right)} \\
& =\frac{1}{m} \sum_{j=1}^{m}\left(\frac{\lambda+s_{j}-\zeta}{\lambda}\right)^{i-1} \frac{\left[R\left(\zeta, \frac{\lambda+s_{j}-\zeta}{\lambda}\right)-\zeta \Pi\left(s, \frac{\lambda+s_{j}-\zeta}{\lambda}\right)\right]}{\left(s_{j}-s\right)}
\end{aligned}
$$

The Laplace-Stieltjes transform (37) is a regular function of $\zeta$ if $\Re(\zeta) \geqq 0$ and $\Re(s)>0$. If $\zeta=\zeta_{j}(s)(j=1,2, \cdots, m)$ is that root in $\zeta$ of the equation

$$
\left(\frac{\lambda+s-\zeta}{\lambda}\right)^{m}=\psi(\zeta)
$$

for which

$$
\lambda+s-\zeta_{j}(s)=\lambda \epsilon_{j}\left[\psi\left(\zeta_{j}(s)\right)\right]^{1 / m}
$$

then by (31) $s_{j}\left(\zeta_{j}(s)\right)=s$ and $s_{r}\left(\zeta_{j}(s)\right) \neq s$ if $r \neq j$. Therefore the coefficient of $1 /\left(s_{j}-s\right)$ on the right side of (37) must vanish if $\zeta=\zeta_{j}(s)=s+\lambda\left[1-\gamma_{j}(s)\right]$, i.e.,

$$
\left\{s+\lambda\left[1-\gamma_{j}(s)\right]\right\} \Pi\left(s, \gamma_{j}(s)\right)=R\left(s+\lambda\left[1-\gamma_{j}(s)\right], \gamma_{j}(s)\right)
$$

if $j=1,2, \cdots, m$. The function $\Pi(s, z)$ in $z$ is a polynomial of degree $m-1$ and by using the Lagrange interpolation formula we obtain from (38) that

$$
\Pi(s, z)=\sum_{j=1}^{m} \frac{R\left(s+\lambda\left[1-\gamma_{j}(s)\right], \gamma_{j}(s)\right)}{s+\lambda\left[1-\gamma_{j}(s)\right]} \prod_{r \neq j}\left(\frac{z-\gamma_{r}(s)}{\gamma_{j}(s)-\gamma_{r}(s)}\right) .
$$

This proves (21). Using the representation (32) we obtain from (29) that

$$
\Omega_{i}(t, \zeta)=\frac{1}{m} \sum_{j=1}^{m} \sum_{k=1}^{m}\left(\frac{\lambda+s_{j}-\zeta}{\lambda}\right)^{k-i}\left[e^{\delta_{j} t} \hat{\Omega}_{k}(\zeta)-\zeta \int_{0}^{t} e^{\delta_{j}(t-u)} P_{k-1}(u) d u\right]
$$

and finally (19) can be obtained by (13). This completes the proof of the theorem.

REMARK 2. By (13) and (37) it follows that

$$
\begin{aligned}
\int_{0}^{\infty} e^{-s t} \Omega(t, \zeta) d t= & \frac{\lambda[1-\psi(\zeta)]}{m \psi(\zeta)} \sum_{j=1}^{m} \frac{\epsilon_{j}[\psi(\zeta)]^{1 / m}}{\left(\zeta-s_{j}\right)\left(s-s_{j}\right)} \\
& \cdot\left[R\left(\zeta, \epsilon_{j}[\psi(\zeta)]^{1 / m}\right)-\zeta \Pi\left(s, \epsilon_{j}[\psi(\zeta)]^{1 / m}\right)\right]
\end{aligned}
$$

where $s_{j}$ is defined by (20) and $\Pi(s, z)$ is given by (39).

Further, we remark that if the initial condition is $\xi(0)=0$ then $\eta(0)=0$ and $R(s, z)=1$. In this case 


$$
\Pi(s, z)=\frac{1}{s+\lambda(1-z)}\left\{1-\prod_{j=1}^{m}\left(\frac{\lambda\left[z-\gamma_{j}(s)\right]}{s+\lambda\left[1-\gamma_{j}(s)\right]}\right)\right\} .
$$

This follows immediately from (38). Now we have

$$
[s+\lambda(1-z)] \Pi(s, z)-1=C(s) \prod_{j=1}^{m}\left[z-\gamma_{j}(s)\right]
$$

because the left side vanishes if $z=\gamma_{j}(s)(j=1,2, \cdots, m)$. The constant $C(s)$ can be obtained by the substitution $z=(\lambda+s) / \lambda$.

If specifically $\xi(+0)=m$ and $t=0$ is a departure point then $P\{\eta(0) \leqq x\}$ $=H(x)$ and $R(s, z)=\psi(s)$. In this case

$$
\Pi(s, z)=\frac{1}{s+\lambda(1-z)}\left\{z^{m}-\prod_{j=1}^{m}\left(\frac{z-\gamma_{j}(s)}{1-\frac{\lambda}{\lambda+s} \gamma_{j}(s)}\right)\right\}
$$

REMARK 3. The above method can be applied word for word to determine the distribution of the virtual waiting time in the case of cyclic queues. In this case we suppose that the inter-arrival times $\left\{\theta_{n}\right\}$ and the service times $\left\{\chi_{n}\right\}$ are independent sequences of independent positive random variables with distribution functions

$$
P\left\{\theta_{n} \leqq x\right\}=\left\{\begin{array}{cl}
1-e^{-\lambda_{j} x} & \text { if } x \geqq 0 \\
0 & \text { if } x<0
\end{array}\right.
$$

and

$$
P\left\{\chi_{n} \leqq x\right\}=H_{j}(x) \quad(j=1,2, \cdots, m),
$$

when $n \equiv j(\bmod m)$. In determining the Laplace-Stieltjes transform of the virtual waiting time we obtain again the matrix equation (28) in which

$$
\boldsymbol{A}=\left\|\begin{array}{ccccc}
\left(\zeta-\lambda_{1}\right) & 0 & \cdots & 0 & \lambda_{m} \psi_{m}(\zeta) \\
\lambda_{1} \psi_{1}(\zeta) & \left(\zeta-\lambda_{2}\right) & \cdots & 0 & 0 \\
\cdot & \cdot & \cdots & \cdot & \cdot \\
0 & 0 & \cdots \lambda_{m-1} \psi_{m-1}(\zeta) & \left(\zeta-\lambda_{m}\right)
\end{array}\right\|
$$

where $\psi_{j}(s)$ is the Laplace-Stieltjes transform of $H_{j}(x)$. In this case the characteristic equation of $\boldsymbol{A}$ is:

$$
|\boldsymbol{A}-s I|=\left(\zeta-\lambda_{1}-s\right) \cdots\left(\zeta-\lambda_{m}-s\right)-\lambda_{1} \cdots \lambda_{m} \psi_{1}(\zeta) \cdots \psi_{m}(\zeta)=0
$$

4. The probability that the server is idle. First of all we shall prove the following auxiliary theorem which we shall need in the sequel. 
Lemma 3. The limit $\lim _{t \rightarrow \infty} P_{j}(t)=P_{j}^{*}(j=0,1,2, \cdots)$ always exists and is independent of the initial state.

Proof. By the theorem of total probability we can write for $k=0,1, \cdots$, $m-1$ that

$$
P_{k}(t)=P_{k}(0) e^{-\lambda t}+\int_{0}^{t} e^{-\lambda(t-u)} d N_{k}(u)+\int_{0}^{t} e^{-\lambda(t-u)} d M_{k-1}(u),
$$

where the last term on the right side is zero if $k=0$, and for $k=m+1$, $m+2, \cdots$ that

$$
\begin{aligned}
P_{k}(t)= & \sum_{j=m}^{k} q_{j}(t) e^{-\lambda t} \frac{(\lambda t)^{k-j}}{(k-j) !} \\
& +\sum_{j=m}^{k} \int_{0}^{t}[1-H(t-u)] e^{-\lambda(t-u)} \frac{[\lambda(t-u)]^{k-j}}{(k-j) !} d N_{j}(u) \\
& +\int_{0}^{t}[1-H(t-u)] e^{-\lambda(t-u)} \frac{[\lambda(t-u)]^{k-m}}{(k-m) !} d M_{m-1}(u)
\end{aligned}
$$

where $q_{j}(t)$ is the probability that the initial queue size is $j$ and there is no departure in the time interval $(0, t]$.

In proving (44) we take into consideration that the event $\xi(t)=k(k<m)$ can occur in the following mutually exclusive ways: the initial state is $E_{k}$ and there is no arrival in the time interval $(0, t]$, or at the instant $u$ (where $0 \leqq u \leqq t)$ there occurs a transition $E_{k+m} \rightarrow E_{m}$ or $E_{k-1} \rightarrow E_{k}$ if $k>0$ and there is no arrival in the time interval $(u, t]$. Finally we get $(44)$ if we keep in mind that the transition $E_{k+m} \rightarrow E_{m}$ and $E_{k-1} \rightarrow E_{k}$ may be the $n$th $(n=1,2, \cdots)$ departure or arrival respectively. Similarly in proving (45) we take into consideration that the event $\xi(t)=k(k \geqq m)$ can occur in the following mutually exclusive ways: the initial state is $E_{j}(j=m, \cdots, k)$ and in the time interval $(0, t]$ there is no departure and $k-j$ customers arrive, or at the instant $u$ (where $0 \leqq u \leqq t)$ there occurs a transition $E_{j+m} \rightarrow E_{j}(j=m, m+1, \cdots, k)$ or a transition $E_{m-1} \rightarrow E_{m}$, the service starting at this instant $u$ does not end in the time interval $(u, t]$ and during this time interval $(u, t]$ respectively $k-j(j=m, m+1, \cdots, k)$ or $k-m$ customers arrive. Finally, we get (45) if we keep in mind that the transition $E_{j+m} \rightarrow E_{j}$ and $E_{m-1} \rightarrow E_{m}$ may be the $n$th $(n=1,2, \cdots)$ departure or arrival respectively.

The transitions $E_{m+j} \rightarrow E_{j}(j=0,1,2, \cdots)$ and similarly the transitions $E_{j} \rightarrow E_{j+1}(j=0,1, \cdots, m-1)$ form a recurrent process. The distances between successive transitions are identically distributed independent random variables having nonlattice distributions. Therefore by a theorem of $\mathrm{D}$. Blackwell [1] it follows that the following limits exist for every $h>0$ and agree with the respective right hand sides: 


$$
\lim _{t \rightarrow \infty} \frac{N_{j}(t+h)-N_{j}(t)}{h}=\lim _{t \rightarrow \infty} \frac{N_{j}(t)}{t} \quad(j=0,1,2, \cdots)
$$

and

$$
\lim _{t \rightarrow \infty} \frac{M_{j}(t+h)-M_{j}(t)}{h}=\lim _{t \rightarrow \infty} \frac{M_{j}(t)}{t} \quad(j=0,1, \cdots, m-1) .
$$

Furthermore these limits are independent of the initial condition.

Forming the Riemann-Stieltjes sums approximating the integrals (44) and (45) respectively and using the relations (46) and (47) we obtain that the $\operatorname{limit}_{\lim _{t \rightarrow \infty}} P_{j}(t)=P_{j}^{*}(j=0,1,2, \cdots)$ always exists irrespective of the initial state.

Specifically we have for $k=0,1, \cdots, m-1$

$$
P_{k}^{*}=\frac{1}{\lambda}\left(N_{k}+M_{k-1}\right)
$$

and for $k=m, m+1, \cdots$

$$
\begin{aligned}
P_{k}^{*}= & \sum_{j=m}^{k} N_{j} \int_{0}^{\infty}[1-H(x)] e^{-\lambda x} \frac{(\lambda x)^{k-j}}{(k-j) !} d x \\
& +M_{m-1} \int_{0}^{\infty}[1-H(x)] e^{-\lambda x} \frac{(\lambda x)^{k-m}}{(k-m) !} d x
\end{aligned}
$$

where we used the notation

$$
\lim _{t \rightarrow \infty} \frac{N_{j}(t)}{t}=N_{j} \text { and } \quad \lim _{t \rightarrow \infty} \frac{M_{j}(t)}{t}=M_{j}
$$

Now let us denote by $Q(t)$ the probability that the server is idle at the instant $t$. Clearly $Q(t)=P\{\xi(t)<m\}$, i.e.,

$$
Q(t)=\sum_{j=0}^{m-1} P_{j}(t)
$$

Hence for $\Re(s)>0$

$$
\int_{0}^{\infty} e^{-s t} Q(t) d t=\sum_{j=0}^{m-1} \Pi_{j}(s)=\Pi(s, 1)
$$

where $\Pi(s, z)$ is given by (21).

If specifically $\xi(0)=0$ then by (42)

$$
\int_{0}^{\infty} e^{-s t} Q(t) d t=\frac{1}{s}\left\{1-\prod_{j=1}^{m}\left(\frac{\lambda\left[1-\gamma_{j}(s)\right]}{s+\lambda\left[1-\gamma_{j}(s)\right]}\right)\right\} .
$$


ThEOREM 2. If $\lambda \alpha<m$ then

$$
\lim _{t \rightarrow \infty} Q(t)=1-\frac{\lambda \alpha}{m}
$$

and if $\lambda \alpha \geqq m$ then

$$
\lim _{t \rightarrow \infty} Q(t)=0
$$

irrespective of the initial condition.

Proof. Referring to Lemma 3 it is sufficient to restrict ourselves to the case when initially the server is idle. Let us denote by $\rho$ the expected number of services in a busy period (possibly $\rho=\infty$ ). The starting points of the busy periods agree with the transitions $E_{m-1} \rightarrow E_{m}$. It is easy to see that the expectation of the distance between two successive transitions $E_{m-1} \rightarrow E_{m}$ is $m \rho / \lambda$. Hence by the theorem of D. Blackwell [1] it follows that for every $h>0$

$$
\lim _{t \rightarrow \infty} \frac{M_{m-1}(t+h)-M_{m-1}(t)}{h}=\lim _{t \rightarrow \infty} \frac{M_{m-1}(t)}{t}=\frac{\lambda}{m \rho}
$$

irrespective of the initial state. We note that $\rho<\infty$ if $\lambda \alpha<m$ and $\rho=\infty$ if $\lambda \alpha \geqq m$. This can be seen as follows. If a busy period terminates, not more than $m$ customers may arrive before the beginning of the next one. Consequently we have the inequality

$$
\rho \alpha<\rho \frac{m}{\lambda} \leqq \rho \alpha+\frac{m}{\lambda}
$$

whence $\rho \leqq 1 /(1-\lambda \alpha / m)$ if $\lambda \alpha<m$ and $\rho=\infty$ if $\lambda \alpha \geqq m$.

If $G(x)$ denotes the distribution function of the length of the busy period then we can write that

$$
Q(t)=1-\int_{0}^{t}[1-G(t-u)] d M_{m-1}(u) .
$$

If $\rho<\infty$ then obviously

$$
\int_{0}^{\infty}[1-G(x)] d x=\rho \alpha
$$

and by using (54) we obtain from (55) that

$$
\lim _{t \rightarrow \infty} Q(t)=1-\frac{\lambda \alpha}{m}
$$

irrespective of $\rho$.

Since evidently 


$$
\left|M_{k}(t)-\sum_{j=0}^{k} N_{j}(t)\right| \leqq 1 \quad(k=0,1, \cdots, m-1)
$$

holds for all $t \geqq 0$ we get by (54) that in case $\rho=\infty$

$$
\lim _{t \rightarrow \infty} \frac{N_{k}(t)}{t}=\lim _{t \rightarrow \infty} \frac{M_{k}(t)}{t}=0 \quad(k=0,1,2, \cdots, m-1)
$$

and by (44)

$$
\lim _{t \rightarrow \infty} P_{k}(t)=0 \quad(k=0,1, \cdots, m-1)
$$

irrespective of the initial state. Hence

$$
\lim _{t \rightarrow \infty} Q(t)=0
$$

if $\rho=\infty$. This completes the proof of the theorem.

REMARK 4. We have seen that $\lim _{t \rightarrow \infty} Q(t)=Q^{*}$ always exists irrespective of the initial state. In this case obviously

$$
\lim _{t \rightarrow \infty} \frac{1}{t} \int_{0}^{t} Q(u) d u=Q^{*}
$$

also holds. Hence we can conclude that

$$
\lim _{s \rightarrow 0} s \int_{0}^{\infty} e^{-s t} Q(t) d t=Q^{*}
$$

Thus by (51) we get

$$
Q^{*}=1-\lim _{s \rightarrow 0} \frac{\lambda\left[1-\gamma_{m}(s)\right]}{s+\lambda\left[1-\gamma_{m}(s)\right]}
$$

because $\left|\gamma_{j}(0)\right|<1$ if $j=1,2, \cdots, m-1$. If now $\left|\gamma_{m}(0)\right|<1$ then $Q^{*}=0$. If $\gamma_{m}(0)=1$ and $\lambda \alpha \leqq m$ then by (8) we get

$$
Q^{*}=\frac{1}{1-\lambda \gamma_{m}^{\prime}(0)}=1-\frac{\lambda \alpha}{m} \text {. }
$$

Since we know that $Q^{*}=1-\lambda \alpha / m$ if $\lambda \alpha<m$ therefore $\gamma_{m}(0)=1$ must hold if $\lambda \alpha<m$.

Finally we prove

Theorem 3. If $\lambda \alpha \geqq m$ then $P_{k}^{*}=0(k=0,1, \cdots, m-1)$ and if $\lambda \alpha<m$ then

$$
\Pi^{*}(z)=\sum_{k=0}^{m-1} P_{k}^{*} z^{k}=\left(1-\frac{\lambda \alpha}{m}\right) \prod_{j=1}^{m-1}\left(\frac{z-\omega_{j}}{1-\omega_{j}}\right) .
$$


Proof. If $\lambda \alpha \geqq m$ then $\rho=\infty$ and by the preceding $P_{k}^{*}=0$ $(k=0,1, \cdots, m-1)$. If $\lambda \alpha<m$ then, $P_{k}^{*}$ being independent of the initial state, we get by (42) that

$$
\sum_{k=0}^{m-1} P_{k}^{*} z^{k}=\lim _{s \rightarrow 0} s \Pi(s, z)=\left(1-\frac{\lambda \alpha}{m}\right) \prod_{j=1}^{m-1}\left(\frac{z-\omega_{j}}{1-\omega_{j}}\right)
$$

which proves (58).

5. The asymptotic behavior of the process $\{\eta(t)\}$.

Theorem 4. If $\lambda \alpha<m$ then the limiting distribution $\lim _{t \rightarrow \infty} W(t, x)=W^{*}(x)$ exists irrespective of the initial distribution. The Laplace-Stieltjes transform of $W^{*}(x)$ is given by

$$
\begin{aligned}
\Omega^{*}(\zeta)= & \int_{0}^{\infty} e^{-\zeta x} d W^{*}(x) \\
= & \left(1-\frac{\lambda \alpha}{m} \frac{[1-\psi(\zeta)]}{m \psi(\zeta)} \sum_{j=1}^{m} \frac{\zeta}{\left\{\zeta-\lambda\left[1-\epsilon_{j}(\psi(\zeta))^{1 / m}\right\}\right\}}\right. \\
& \frac{\left.\epsilon_{j} \mid \psi(\zeta)\right]^{1 / m}}{\left\{1-\epsilon_{j}[\psi(\zeta)]^{1 / m}\right.} \prod_{r=1}^{m-1}\left(\frac{\epsilon_{j}[\psi(\zeta)]^{1 / m}-\omega_{r}}{1-\omega_{r}}\right)
\end{aligned}
$$

where $\omega_{1}, \omega_{2}, \cdots, \omega_{m-1}$ are defined in Lemma 2. If $\lambda \alpha \geqq m$ then $\lim _{t \rightarrow \infty} W(t, x)$ $=0$ for every $x$.

Proof. We shall write

$$
W^{*}(x)=\sum_{=1}^{m} W_{j}^{*}(x)
$$

where

$$
W_{j}^{*}(x)=\lim _{t \rightarrow \infty} W_{j}(t, x)
$$

and

$$
\Omega^{*}(\zeta)=\sum_{j=1}^{m} \Omega_{j}^{*}(\zeta)
$$

where

$$
\Omega_{j}^{*}(\zeta)=\int_{0}^{\infty} e^{-\zeta x} d W_{j}^{*}(x) .
$$

Further we shall use the following vector notation: 


$$
\Omega^{*}(\zeta)=\left\|\begin{array}{c}
\Omega_{1}^{*}(\zeta) \\
\Omega_{2}^{*}(\zeta) \\
\vdots \\
\Omega_{m}^{*}(\zeta)
\end{array}\right\|, \quad P^{*}=\left\|\begin{array}{c}
P_{0}^{*} \\
P_{1}^{*} \\
\vdots \\
P_{m-1}^{*}
\end{array}\right\|
$$

Thus we have

$$
\lim _{t \rightarrow \infty} \mathbf{\Omega}(t, \zeta)=\mathbf{\Omega}^{*}(\zeta)
$$

First consider the case $\lambda \alpha<m$. Then

$$
\lim _{t \rightarrow \infty} P_{j}(t)=P_{j}^{*}>0 \quad(j=0,1, \cdots, m-1)
$$

exists and is independent of the initial state. If we restrict ourselves to imaginary $\zeta$ then by $(40)$ it can be proved that $\lim _{t \rightarrow \infty} \boldsymbol{\Omega}(t, \zeta)=\mathbf{\Omega} *(\zeta)$ exists If $|\zeta|<a$, where $a$ is a sufficiently small positive number, and that $\mathbf{\Omega}^{*}(\zeta)$ is continuous at $\zeta=0$. Hence it follows by a theorem of A. Zygmund [10] that the $\operatorname{limit}_{\lim _{t \rightarrow \infty}} W_{j}(t, x)=W_{j}^{*}(x)$ exists and further that the Laplace-Stieltjes transform of $W_{j}^{*}(x)$ is $\Omega_{j}^{*}(\zeta)=\lim _{t \rightarrow \infty} \Omega_{j}(t, \zeta)$ defined for $\Re(\zeta) \geqq 0$. Thus $\lim _{t \rightarrow \infty} \boldsymbol{\Omega}(t, \zeta)=\boldsymbol{\Omega}^{*}(\zeta)$ also exists and by $(28)$ we get that $\boldsymbol{\Omega}^{*}(\zeta)$ satisfies the equation

$$
A \mathbf{\Omega}^{*}(\zeta)=\zeta P^{*}
$$

Since $|\boldsymbol{A}| \neq 0$ if $\zeta \neq 0$, this equation has one and only one solution if $\zeta \neq 0$. Using the canonical decomposition (32) of $\boldsymbol{A}$ we get explicitly

$$
\begin{aligned}
\Omega_{i}^{*}(\zeta) & =\frac{\zeta}{m} \sum_{k=1}^{m} \sum_{j=1}^{m}\left(\frac{\lambda+s_{j}-\zeta}{\lambda}\right)^{k-i} \frac{P_{k-1}^{*}}{s_{j}} \\
& =\frac{\zeta}{m} \sum_{j=1}^{m} \frac{1}{s_{j}}\left(\frac{\lambda}{\lambda+s_{j}-\zeta}\right)^{i-1} \Pi^{*}\left(\frac{\lambda+s_{j}-\zeta}{\lambda}\right)
\end{aligned}
$$

where $s_{j}$ is defined by (20) and $\mathrm{II}^{*}(z)$ by (58), whence for $\zeta \neq 0$

$$
\begin{aligned}
\Omega^{*}(\zeta) & =\sum_{i=1}^{m} \Omega_{i}^{*}(\zeta) \\
& =\frac{\zeta[1-\psi(\zeta)\rfloor}{m \psi(\zeta)} \sum_{j=1}^{m} \frac{\epsilon_{j}[\psi(\zeta)]^{1 / m}}{\left\{1-\epsilon_{j}[\psi(\zeta)]^{1 / m}\right\}} \frac{\Pi^{*}\left(\epsilon_{j}[\psi(\zeta)]^{1 / m}\right)}{\left\{\zeta-\lambda\left[1-\epsilon_{j}(\psi(\zeta))^{1 / m}\right]\right\}}
\end{aligned}
$$

and clearly $\Omega^{*}(0)=1$. This proves (59).

If $\lambda \alpha \geqq m$ then $P_{j}^{*}=0 \quad(j=0,1, \cdots, m-1)$ and $\lim _{t \rightarrow \infty} \boldsymbol{\Omega}(t, \zeta)=0$ for $\Re(\zeta) \geqq 0$, whence $\lim _{t \rightarrow \infty} W(t, x)=0$. This completes the proof of the theorem. 
6. The transient behavior of the process $\left\{\eta_{n}\right\}$. Let us define $\theta_{n}=\tau_{(n+1) m}$ $-\tau_{n m}(n=1,2, \cdots)$; then obviously

$$
\eta_{n+1}=\left[\eta_{n}+\chi_{n}-\theta_{n}\right]^{+}
$$

where $[a]^{+}=\max (a, 0)$. Here $\left\{\chi_{n}\right\}$ and $\left\{\theta_{n}\right\}$ are independent sequences of identically distributed, independent random variables with distribution functions $P\left\{\chi_{n} \leqq x\right\}=H(x)$ and $P\left\{\theta_{n} \leqq x\right\}=F_{m}(x)$ defined by (1).

We need

Lemma 4. Let $\xi$ and $\theta$ be non-negative, independent random variables for which $P\{\theta \leqq x\}=F_{m}(x)$ defined by (1). Define

$$
\Phi(s)=E\left\{e^{-8 \xi}\right\} .
$$

If $\Re(s) \geqq 0$ then we have

$$
\begin{aligned}
& E\left\{e^{-s[\xi-\theta]^{+}}\right\} \\
& = \begin{cases}\frac{\lambda^{m} \Phi(s)-\sum_{j=0}^{m-1} \frac{(-1)^{j} \Phi^{(j)}(\lambda)}{j !}\left[\lambda^{m}(\lambda-s)^{j}-\lambda^{j}(\lambda-s)^{m}\right]}{(\lambda-s)^{m}} & \text { if } s \neq \lambda, \\
\sum_{j=0}^{m} \frac{(-1)^{j} \Phi^{(j)}(\lambda)}{j !} & \text { if } s=\lambda .\end{cases}
\end{aligned}
$$

Proof. We have

$$
\begin{aligned}
E\left\{e^{-s[\xi-\theta]^{+}} \mid \xi=x\right\}=\sum_{j=0}^{m-1} e^{-\lambda x} \frac{(\lambda x)^{j}}{j !}+\int_{0}^{x} e^{-8(x-y)} e^{-\lambda y} \frac{(\lambda y)^{m-1}}{(m-1) !} \lambda d y \\
= \begin{cases}\frac{\lambda^{m} e^{-s x}-\sum_{j=0}^{m-1} e^{-\lambda x} \frac{x^{j}}{j !}\left[\lambda^{m}(s-\lambda)^{j}-\lambda^{j}(s-\lambda)^{m}\right]}{(\lambda-s)^{m}} & \text { if } s \neq \lambda, \\
\sum_{j=0}^{m} e^{-\lambda x} \frac{(\lambda x)^{j}}{j !} & \text { if } s=\lambda,\end{cases}
\end{aligned}
$$

whence (66) follows.

Theorem 5. Let

$$
\Omega_{n}(s)=E\left\{e^{-s \eta_{n}}\right\} \quad(n=1,2, \cdots) .
$$

If, in particular $\eta_{1} \equiv 0$, then we have for $\Re(s) \geqq 0$ and $|w|<1$ that

$$
\sum_{n=1}^{\infty} \Omega_{n}(s) w^{n-1}=\frac{\lambda^{m} \prod_{r=1}^{m}\left(1-\frac{s}{\lambda\left[1-g_{r}(w)\right]}\right)}{(\lambda-s)^{m}-w \lambda^{m} \psi(s)}
$$


where $z=g_{r}(w)(r=1,2, \cdots, m)$ are the $m$ roots in $z$ of the equation

$$
z^{m}=\operatorname{w\psi }(\lambda(1-z))
$$

in the unit circle $|z|<1$.

Proof. By (64) and (66) we can write for $s \neq \lambda$ that

$$
\Omega_{n+1}(s)=\frac{\lambda^{m} \psi(s) \Omega_{n}(s)-s C_{m-1}(s)}{(\lambda-s)^{m}}
$$

where $C_{m-1}(s)$ is in $s$ a polynomial of degree $m-1$. Hence

$$
\sum_{n=1}^{\infty} \Omega_{n}(s) w^{n-1}=\frac{(\lambda-s)^{m} \Omega_{1}(s)-s C_{m-1}(s, w)}{(\lambda-s)^{m}-w \lambda^{m} \psi(s)}
$$

where $C_{m-1}(s, w)$ is in $s$ also a polynomial of degree $m-1$. The left hand side of $(71)$ is a regular function of $s$ if $\Re(s) \geqq 0$ and $|w|<1$. In this domain the denominator of the right hand side of $(71)$ has $m$ roots

$$
s=\lambda\left[1-g_{r}(w)\right] \quad(r=1,2, \cdots, m) .
$$

These must be also roots of the numerator. Therefore the polynomial $C_{m-1}(s, w)$ is determined uniquely. If $w \neq 0$ then these roots are distinct and by the Lagrange interpolation formula

$$
C_{m-1}(s, w)=\sum_{r=1}^{m} \frac{\Omega_{1}\left(\lambda\left[1-g_{r}(w)\right]\right)\left[g_{r}(w)\right]^{m}}{\left[1-g_{r}(w)\right]} \prod_{\nu \neq r}\left\{\frac{s-\lambda\left[1-g_{\nu}(w)\right]}{g_{\nu}(w)-g_{r}(w)}\right\} .
$$

If, in particular $\eta_{1} \equiv 0$, i.e., $\Omega_{1}(s) \equiv 1$, then we obtain immediately that

$$
C_{m-1}(s, w)=\frac{1}{s}\left\{(\lambda-s)^{m}-\lambda^{m} \prod_{r=1}^{m}\left(1-\frac{s}{\lambda\left[1-g_{r}(w)\right]}\right)\right\} .
$$

If $w=0$ then (71) reduces to $\Omega_{1}(s)$. This proves (69).

7. The limiting behavior of the process $\left\{\eta_{n}\right\}$. Let

$$
P\left\{\eta_{n} \leqq x\right\}=W_{n}(x) \text {. }
$$

Now we shall prove the following theorem which is a particular case of a more general theorem of F. Pollaczek [7].

Theorem 6. If $\lambda \alpha<m$ then the limiting distribution $\lim _{n \rightarrow \infty} W_{n}(x)=W(x)$ exists and is independent of the initial distribution. The Laplace-Stieltjes transform of $W(x)$ is

$$
\Omega(s)=\int_{0}^{\infty} e^{-s x} d W(x)=\left(1-\frac{\lambda \alpha}{m}\right) \frac{m s \lambda^{m-1} \prod_{r=1}^{m-1}\left(1-\frac{s}{\lambda\left(1-\omega_{r}\right)}\right)}{\lambda^{m} \psi(s)-(\lambda-s)^{m}}
$$


where $\omega_{1}, \omega_{2}, \cdots, \omega_{m-1}$ are the $m-1$ roots in $z$ of the equation

$$
z^{m}=\psi(\lambda(1-z))
$$

in the unit circle $|z|<1$. If $\lambda \alpha \geqq m$ then $\lim _{n \rightarrow \infty} W_{n}(x)=0$ for every $x$.

Proof. The statement concerning the existence of the limiting distribution is a consequence of a theorem of D. V. Lindley [5]. It remains only to find the explicit form of $\Omega(s)$ in the case $\lambda \alpha<m . \Omega(s)$ is independent of the initial distribution. If we suppose that $\eta_{1} \equiv 0$ then from (69) by using Abel's theorem it follows that

$$
\Omega(s)=\lim _{w \rightarrow 1}(1-w) \sum_{n=1}^{\infty} \Omega_{n}(s) w^{n-1}=\left(1-\frac{\lambda \alpha}{m}\right) \frac{m s \lambda^{m-1} \prod_{r=1}^{m}\left(1-\frac{s}{\lambda\left(1-\omega_{r}\right)}\right)}{\lambda^{m} \psi(s)-(\lambda-s)^{m}}
$$

because $g_{m}^{\prime}(1)=1 /(m-\lambda \alpha)$.

REMARK 5. If $\lambda \alpha=m$ then $\Omega(s) \equiv 0$ and therefore it is impossible that $\left|\omega_{r}\right|<1(r=1,2, \cdots, m)$. This proves that $\omega_{m}=1$ if $\lambda \alpha=m$.

8. The transient behavior of the queue size. It is easy to see that the sequence of random variables $\left\{\xi_{n}\right\}$ forms a homogeneous Markov chain, namely we have

$$
\xi_{n+1}=\left[\xi_{n}-m\right]++\nu_{n+1}
$$

where $\nu_{n}$ denotes the number of customers arriving during the $n$th service. The $\left\{\nu_{n}\right\}$ is a sequence of identically distributed, independent random variables for which

$$
P\left\{\nu_{n}=j \mid \chi_{n}=x\right\}=e^{-\lambda x} \frac{(\lambda x)^{j}}{j !}
$$

and unconditionally

$$
P\left\{\nu_{n}=j\right\}=\int_{0}^{\infty} e^{-\lambda x} \frac{(\lambda x)^{j}}{j !} d H(x)
$$

Write

$$
p_{i k}^{(n)}=P\left\{\xi_{n}=k \mid \xi_{0}=i\right\} .
$$

TheOREM 7. If $|z| \leqq 1$ and $|w|<1$ then

$$
\sum_{n=0}^{\infty} \sum_{k=0}^{\infty} p_{0 k}^{(n)} w^{n k} z^{k}=1-\frac{\operatorname{w\psi }(\lambda(1-z)) \prod_{r=1}^{m}\left(\frac{z-g_{r}(w)}{1-g_{r}(w)}\right)}{\operatorname{w\psi } \psi(\lambda(1-z))-z^{m}}
$$

where $z=g_{r}(w)(r=1,2, \cdots, m)$ are the $m$ roots in $z$ of the equation 


$$
z^{m}=\operatorname{urt}(\lambda(1-z))
$$

in the unit circle $|z|<1$.

Instead of proving this theorem we shall prove the following more general theorem, from which it can be obtained as a particular case.

TheOREM 8. Let us define

$$
U_{n}(s, z)=E\left\{e^{-s \tau_{n}{ }^{\prime} z^{\xi_{n}}}\right\},
$$

for $\Re(s) \geqq 0$ and $|z| \leqq 1$. If we suppose that $\xi(0)=0$, then we have for $\Re(s) \geqq 0$, $|z| \leqq 1$ and $|w|<1$ that

$$
\sum_{n=1}^{\infty} U_{n}(s, z) w^{n}=\frac{w \psi(s+\lambda(1-z)) \prod_{r=1}^{m}\left(\frac{\lambda\left[z-\gamma_{r}(s, w)\right.}{s+\lambda\left[1-\gamma_{r}(s, w)\right]}\right)}{z^{m}-\operatorname{w\psi }(s+\lambda(1-z))}
$$

where $z=\gamma_{r}(s, w)(r=1,2, \cdots, m)$ are the $m$ roots in $z$ of the equation

$$
z^{m}=\operatorname{req}(s+\lambda(1-z))
$$

in the unit circle $|z|<1$.

Proof. Now we can write that

$$
\xi_{n+1}=\left[\xi_{n}-m\right]++\nu_{n+1}
$$

and

$$
\tau_{n+1}^{\prime}=\tau_{n}^{\prime}+\chi_{n+1}+\sum_{j=1}^{\left[m-\xi_{n}\right]^{+}} \vartheta_{n+1}^{(j)}
$$

where $\left\{\chi_{n}\right\}(n=1,2, \cdots)$ and $\left\{\vartheta_{n}^{(j)}\right\}(n=1,2, \cdots ; j=1,2, \cdots, m)$ are independent sequences of identically distributed, independent random variables with distribution functions $P\left\{\chi_{n} \leqq x\right\}=H(x)$ and $P\left\{\vartheta_{n}^{(j)} \leqq x\right\}=F(x)$ defined by (2). The random variable $\nu_{n}$ depends only on $\chi_{n}$ and its distribution is given by (78).

Since

$$
E\left\{e^{-s x_{n} z^{\nu_{n}}}\right\}=\psi(s+\lambda(1-z))
$$

we get by (85) and (86) that

$$
U_{n+1}(s, z)=\psi(s+\lambda(1-z))\left\{\frac{U_{n}(s, z)-\sum_{j=0}^{m-1} C_{n j}(s) z^{3}}{z^{m}}+\sum_{j=0}^{m-1} C_{n j}(s)\left(\frac{\lambda}{\lambda+s}\right)^{m-j}\right\}
$$

where 


$$
C_{n j}(s)=P\left\{\xi_{n}=j\right\} E\left\{e^{-8 \tau_{n}^{\prime}} \mid \xi_{n}=j\right\}
$$

Hence

$$
\sum_{n=0}^{\infty} U_{n}(s, z) w^{n}=\frac{z^{m} U_{0}(s, z)-w \psi(s+\lambda(1-z)) C_{m}(z, s, w)}{z^{m}-w \psi(s+\lambda(1-z))}
$$

where

$$
C_{m}(z, s, w)=\sum_{n=0}^{\infty} \sum_{j=0}^{m-1} C_{n j}(s) w^{n}\left[z^{j}-z^{m}\left(\frac{\lambda}{\lambda+s}\right)^{m-j}\right]
$$

is in $z$ a polynomial of degree $m$ and $C_{m}(z, s, w)$ vanishes if $z=(\lambda+s) / \lambda$.

The left side of (87) is a regular function of $z$ if $|z| \leqq 1, \Re(s) \geqq 0$ and $|w|<1$. In this domain the denominator of the right hand side of (87) has exactly $m$ roots $z=\gamma_{r}(s, w)(r=1,2, \cdots, m)$. These must be also roots of the numerator. Thus the polynomial $C_{m}(z, s, w)$ is determined uniquely. If we exclude the trivial case $w=0$ then the roots $\gamma_{r}(s, w)$ are distinct and $C_{m}(z, s, w)$ can be obtained explicitly by the Lagrange interpolation formula

$$
C_{m}(z, s, w)=\sum_{r=1}^{m} \frac{U_{0}\left(s, \gamma_{r}(s, w)\right)[s+\lambda(1-z)]}{s+\lambda\left[1-\gamma_{r}(s, w)\right]} \prod_{\nu \neq r}\left(\frac{z-\gamma_{\nu}(s, w)}{\gamma_{r}(s, w)-\gamma_{\nu}(s, w)}\right)
$$

If, in particular $\tau_{0}^{\prime}=0$ and $\xi_{0}=m$, then $U_{0}(s, z)=z^{m}$ and we obtain immediately that

$$
C_{m}(z, s, w)=z^{m}-\prod_{r=1}^{m}\left(\frac{z-\gamma_{r}(s, w)}{1-\frac{\lambda}{\lambda+s} \gamma_{r}(s, w)}\right) .
$$

If $\xi(0)=0$ then $U_{n}(s, z)(n=1,2, \cdots)$ obviously agrees with the $U_{n}(s, z)$ calculated by the assumption $U_{0}(s, z)=(\lambda /(\lambda+s))^{m} z^{m}$. In this case we obtain immediately that

$$
C_{m}(z, s, w)=\left(\frac{\lambda z}{\lambda+s}\right)^{m}-\prod_{r=1}^{m}\left(\frac{\lambda\left[z-\gamma_{r}(s, w)\right]}{s+\lambda\left[1-\gamma_{r}(s, w)\right]}\right) .
$$

This proves the theorem. If we write $s=0$ in (83) then we get (80).

REMARK 6. We have the obvious relation

$$
P\left\{\xi_{n}=j\right\}=\int_{0}^{\infty} e^{-\lambda x} \frac{(\lambda x)^{j}}{j !} d\left[W_{n}(x) * H(x)\right]
$$

whence

$$
E\left\{z^{\xi_{n}}\right\}=\Omega_{n}(\lambda(1-z)) \psi(\lambda(1-z)) .
$$

Now using (69) we can prove (80) also in this way. 
Theorem 9. Let

$$
\Pi_{k}(s)=\int_{0}^{\infty} e^{-s t} P_{k}(t) d t
$$

for $\Re(s)>0$. If we suppose that $\xi(0)=0$ then we have for $\Re(s)>0$ and $|z| \leqq 1$ that

$$
\begin{aligned}
\sum_{k=0}^{\infty} \Pi_{k}(s) z^{k}= & \frac{1}{s+\lambda(1-z)} \\
& \cdot\left\{1-\frac{\left(1-z^{m}\right) \psi(s+\lambda(1-z))}{z^{m}-\psi(s+\lambda(1-z))} \prod_{r=1}^{m}\left(\frac{\lambda\left[z-\gamma_{r}(s)\right]}{s+\lambda\left[1-\gamma_{r}(s)\right]}\right)\right\}
\end{aligned}
$$

where $z=\gamma_{r}(s)(r=1,2, \cdots, m)$ are the $m$ roots in $z$ of the equation

$$
z^{m}=\psi(s+\lambda(1-z))
$$

in the unit circle $|z|<1$.

Proof. Let us form the Laplace transforms of (44) and (45) in this special case; then we get easily that

$$
\sum_{k=0}^{m-1} \Pi_{k}(s) z^{k}=\frac{1}{\lambda+s}\left\{1+\sum_{j=0}^{m-1} z^{j} \int_{0}^{\infty} e^{-s t} d N_{j}(t)+z \sum_{j=0}^{m-2} z^{j} \int_{0}^{\infty} e^{-s t} d M_{j}(t)\right\}
$$

and

$$
\begin{aligned}
\sum_{k=m}^{\infty} \Pi_{k}(s) z^{k}= & \frac{1-\psi(s+\lambda(1-z))}{s+\lambda(1-z)} \\
& \cdot\left\{\sum_{j=m}^{\infty} z^{j} \int_{0}^{\infty} e^{-s t} d N_{j}(t)+z^{m} \int_{0}^{\infty} e^{-s t} d M_{m-1}(t)\right\} .
\end{aligned}
$$

Since clearly

$$
N_{j}(t)=\sum_{n=1}^{\infty} P\left\{\tau_{n}^{\prime} \leqq t, \xi_{n}=j\right\}
$$

we obtain by (83) that

$$
\sum_{j=0}^{\infty} z^{j} \int_{0}^{\infty} e^{-s t} d N_{j}(t)
$$

$$
=\sum_{n=1}^{\infty} U_{n}(s, z)=\frac{\psi(s+\lambda(1-z)) \prod_{r=1}^{m}\left(\frac{\lambda\left[z-\gamma_{r}(s)\right]}{s+\lambda\left[1-\gamma_{r}(s)\right]}\right)}{z^{m}-\psi(s+\lambda(1-z))} .
$$

Further, since 


$$
M_{j}(t)=\lambda \int_{0}^{t} P_{j}(u) d u \quad(j=0,1,2, \cdots)
$$

we get by (42) that

$$
\begin{aligned}
\sum_{j=0}^{m-1} z^{j} \int_{0}^{\infty} e^{-s t} d M_{j}(t)=\lambda \Pi(s, z)= & \frac{\lambda}{s+\lambda(1-z)} \\
& \left\{1-\prod_{r=1}^{m}\left(\frac{\lambda\left[z-\gamma_{r}(s)\right]}{s+\lambda\left[1-\gamma_{r}(s)\right]}\right)\right\} .
\end{aligned}
$$

From (101) we get

$$
\int_{0}^{\infty} e^{-s t} d M_{m-1}(t)=\prod_{j=1}^{m}\left(\frac{\lambda}{s+\lambda\left[1-\gamma_{r}(s)\right]}\right)
$$

and from (96) and (101)

$$
\begin{aligned}
& \sum_{j=0}^{m-1} z^{j} \int_{0}^{\infty} e^{-s t} d N_{j}(t) \\
&=\prod_{j=1}^{m}\left(\frac{\lambda z}{s+\lambda\left[1-\gamma_{r}(s)\right]}\right)-\prod_{j=1}^{m}\left(\frac{\lambda\left[z-\gamma_{r}(s)\right]}{s+\lambda\left[1-\gamma_{r}(s)\right]}\right) .
\end{aligned}
$$

Comparing the above formulas we obtain (94) which was to be proved.

\section{The limiting distribution of the queue size.}

THEOREM 10. If $\lambda \alpha<m$ then the limiting distribution $\lim _{n \rightarrow \infty} P\left\{\xi_{n}=k\right\}$ $=P_{k}(k=0,1,2, \cdots)$ exists and is independent of the initial distribution. We have

$$
\sum_{k=0}^{\infty} P_{k} z^{k}=m\left(1-\frac{\lambda \alpha}{m}\right) \frac{(1-z) \psi(\lambda(1-z)) \prod_{r=1}^{m-1}\left(\frac{z-\omega_{r}}{1-\omega_{r}}\right)}{z^{m}-\psi(\lambda(1-z))}
$$

where $\omega_{1}, \omega_{2}, \cdots, \omega_{m-1}$ are the $m-1$ roots in $z$ of the equation

$$
z^{m}=\psi(\lambda(1-z))
$$

in the unit circle $|z|<1$. If $\lambda \alpha \geqq m$ then $\lim _{n \rightarrow \infty} P\left\{\xi_{n}=k\right\}=0$ for every $k$.

Proof. The sequence $\left\{\xi_{n}\right\}$ is an irreducible and aperiodic Markov chain. Therefore $\lim _{n \rightarrow \infty} P\left\{\xi_{n}=k\right\}=P_{k}$ always exists and is independent of the initial distribution. Either every $P_{k}>0$ and $\left\{P_{k}\right\}$ is a probability distribution, or every $P_{k}=0$. By using Abel's theorem we have

$$
\sum_{k=0}^{\infty} P_{k}^{k} z=\lim _{w \rightarrow 1}(1-w) \sum_{n=0}^{\infty} \sum_{k=0}^{\infty} p_{i k}^{(n)} z^{k} w^{n},
$$

and the right hand side can be calculated by (80). This proves (104). 
THEOREM 11. If $\lambda \alpha<m$ then the limiting distribution $\lim _{t \rightarrow \infty} P\{\xi(t)=k\}$ $=P_{k}^{*}(k=0,1,2, \cdots)$ exists and is independent of the initial distribution. We have

$$
P_{k}^{*}= \begin{cases}\frac{1}{m}\left(P_{0}+P_{1}+\cdots+P_{k}\right) & \text { if } k<m, \\ \frac{1}{m}\left(P_{k-m}+P_{k-m+1}+\cdots+P_{k}\right) & \text { if } k \geqq m\end{cases}
$$

where the distribution $\left\{P_{j}\right\}$ is defined by (104). If $\lambda \alpha \geqq m$ then $\lim _{t \rightarrow \infty} P\{\xi(t)=k\}$ $=0$ for every $k$.

Proof. Obviously

$$
M_{k}(t)=\lambda \int_{0}^{t} P_{k}(u) d u .
$$

We have proved that $\lim _{t \rightarrow \infty} P_{k}(t)=P_{k}^{*}(k=0,1,2, \cdots)$ always exists and hence

$$
\lim _{t \rightarrow \infty} \frac{M_{k}(t)}{t}=\lambda P_{k}^{*} .
$$

On the other hand we can see easily that

$$
\left|M_{k}(t)-\sum_{j=k-m+1}^{k} N_{j}(t)\right| \leqq 1
$$

for all $t \geqq 0$. Here $N_{j}(t)=0$ if $j<0$. Thus

$$
P_{k}^{*}=\frac{1}{\lambda} \lim _{t \rightarrow \infty} \frac{M_{k}(t)}{t}=\lim _{t \rightarrow \infty} \frac{1}{\lambda t} \sum_{j=k=m+1}^{k} N_{j}(t) .
$$

Now let us denote by $N(t)$ the expected number of departures occurring in the time interval $(0, t]$. By the theory of Markov chains it follows that

$$
\lim _{t \rightarrow \infty} \frac{N_{j}(t)}{N(t)}=P_{j} \quad(j=0,1,2, \cdots)
$$

and therefore

$$
P_{k}^{*}=\frac{1}{\lambda}\left(\sum_{j=k-m+1}^{k} P_{j}\right) \lim _{t \rightarrow \infty} \frac{N(t)}{t},
$$

where $P_{j}=0$ if $j<0$. If we suppose that $\lambda \alpha<m$ then by (58) and (104) we obtain that $P_{0} / P_{0}^{*}=m$ and hence if we write $k=0$ in (108) we get

$$
\lim _{t \rightarrow \infty} \frac{N(t)}{t}=\frac{\lambda}{m}
$$


and by (108)

$$
P_{k}^{*}=\frac{1}{m} \sum_{j=k-m+1}^{k} P_{j}
$$

for every $k$. If $\lambda \alpha \geqq m$ then every $P_{j}=0$ and since $\lim _{t \rightarrow \infty} N(t) / t$ is evidently finite by (108) $P_{k}^{*}=0$ for every $k$. This completes the proof of the theorem.

10. The stochastic law of the busy period. Now we shall prove the following theorem of B. W. Conolly [2]. A more general theorem has been proved earlier by F. Pollaczek [8].

THEOREM 12. The Laplace-Stieltjes transform

$$
\Gamma_{n}(s)=\int_{0}^{\infty} e^{-8 x} d G_{n}(x)
$$

is given by the generating function

$$
\sum_{n=1}^{\infty} \Gamma_{n}(s) w^{n}=1-\prod_{r=1}^{m}\left[1-\gamma_{r}(s, w)\right]
$$

for $\Re(s) \geqq 0$ and $|w| \leqq 1$ where $\gamma_{r}(s, w)(r=1,2, \cdots, m)$ are defined in Lemma 1.

Proof. Denote by $G_{n k}(x)$ the probability that the busy period consists of at least $n$ services, the total service time of the first $n$ batches is at most $x$, and at the end of the $n$th service $k$ customers are present in the queue. Then evidently

$$
G_{n}(x)=\sum_{k=0}^{m-1} G_{n k}(x)
$$

If we write

$$
\Gamma_{n k}(s)=\int_{0}^{\infty} e^{-s x} d G_{n k}(x)
$$

then

$$
\Gamma_{n}(s)=\sum_{k=0}^{m-1} \Gamma_{n k}(s)
$$

Now by the theorem of total probability we can write that

$$
G_{1 k}(x)=\int_{0}^{x} e^{-\lambda y} \frac{(\lambda y)^{k}}{k !} d H(y)
$$

and 
$G_{n k}(x)=\sum_{r=m}^{m+k} \int_{0}^{x} G_{n-1, r}(x-y) e^{-\lambda y} \frac{(\lambda y)^{k-r+m}}{(k-r+m) !} d H(y) \quad(n=2,3, \cdots)$.

Forming Laplace-Stieltjes transforms we get

$$
\Gamma_{1 k}(s)=\int_{0}^{\infty} e^{-(\lambda+s) y} \frac{(\lambda y)^{k}}{k !} d H(y)
$$

and

$$
\Gamma_{n k}(s)=\sum_{r=m}^{m+k} \Gamma_{n-1, r}(s) \int_{0}^{\infty} e^{-(\lambda+s) y} \frac{(\lambda y)^{k-r+m}}{(k-r+m) !} d H(y) .
$$

If we introduce the generating function

$$
C_{n}(s, z)=\sum_{k=0}^{\infty} \Gamma_{n k}(s) z^{k}
$$

then we have

$$
\begin{gathered}
C_{1}(s, z)=\psi(s+\lambda(1-z)) \\
z^{m} C_{n}(s, z)=\psi(s+\lambda(1-z))\left[C_{n-1}(s, z)-\sum_{r=0}^{m-1} \Gamma_{n-1, r}(s) z^{r}\right] .
\end{gathered}
$$

Hence

$$
\sum_{n=1}^{\infty} C_{n}(s, z) w^{n}=w \psi(s+\lambda(1-z)) \frac{z^{m}-\sum_{n=1}^{\infty} \sum_{r=0}^{m-1} \Gamma_{n, r}(s) z^{r} w^{n}}{z^{m}-w \psi(s+\lambda(1-z))} \cdot
$$

The left hand side of (115) is a regular function of $z$ if $|z| \leqq 1, \Re(s) \geqq 0$ and $|w|<1$. In this domain the denominator of the right hand side has exactly $m$ roots $z=\gamma_{r}(s, w)(r=1,2, \cdots, m)$. These must also be roots of the numerator. Thus the numerator, being a polynomial of degree $m$, is determined uniquely, namely

$$
z^{m}-\sum_{n=1}^{\infty} \sum_{r=0}^{m-1} \Gamma_{n r}(s) z^{r} w^{n}=\prod_{r=1}^{m}\left[z-\gamma_{r}(s, w)\right] .
$$

If $z=1$ in (116) then

$$
\sum_{n=1}^{\infty} \Gamma_{n}(s) w^{n}=1-\prod_{r=1}^{m}\left[1-\gamma_{r}(s, w)\right]
$$

for $|w|<1$ and this is also true for $|w|=1$, which can be shown by analytical continuation. This completes the proof of the theorem.

THEOREM 13. If $G(x)$ denotes the distribution function of the length of the busy period, then we have 


$$
\lim _{x \rightarrow \infty} G(x)=\left\{\begin{array}{cl}
1 & \text { if } \lambda \alpha \leqq m \\
1-\prod_{r=1}^{m}\left(1-\omega_{r}\right) & \text { if } \lambda \alpha>m
\end{array}\right.
$$

where $\omega_{1}, \omega_{2}, \cdots, \omega_{m}$ are the $m$ roots in $z$ of the equation

$$
z^{m}=\psi(\lambda(1-z))
$$

in the unit circle $|z|<1$.

Proof. Let

$$
\Gamma(s)=\int_{0}^{\infty} e^{-s x} d G(x)
$$

be the Laplace-Stieltjes transform of the distribution function of the length of the busy period. By (111) we have

$$
\Gamma(s)=1-\prod_{r=1}^{m}\left[1-\gamma_{r}(s)\right] .
$$

Hence

$$
\lim _{x \rightarrow \infty} G(x)=\lim _{s \rightarrow 0} \Gamma(s)=1-\prod_{r=1}^{m}\left(1-\omega_{r}\right),
$$

where $\omega_{m}=1$ if $\lambda \alpha \leqq m$, as was to be proved.

\section{REFERENCES}

1. D. Blackwell, A renewal theorem, Duke Math. J. vol. 15 (1948) pp. 145-150.

2. B. W. Conolly, The busy period in relation to the queueing process $E_{K} / G / 1$, to be published.

3. A. J. Fabens, The solution of queueing and inventory models, Technical Report No. 20, December 7, 1959. Department of Statistics, Stanford University.

4. R. R. P. Jackson and D. G. Nickols, Some equilibrium results for the queueing process $E_{K} / M / 1$, J. Roy. Statist. Soc. Ser. B. vol. 18 (1956) pp. 275-279.

5. D. V. Lindley, The theory of queues with a single server, Proc. Cambridge Philos. Soc. vol. 48 (1952) pp. 277-289.

6. F. Pollaczek, Über eine Aufgabe der Wahrscheinlichkeitstheorie, Math. Z. vol. 32 (1930) pp. 64-100; 729-750.

7. - Problèmes stochastiques posés par le phênomène de formation d'une queue d'attente ḋ un guichet et par des phénomènes apparentés, Paris, Gauthier-Villars, 1957.

8. - Sur la répartition des périodes d'occupation ininterrompue d'un guichet, C. R. Acad. Sci. Paris vol. 234 (1952) pp. 2042-2044.

9. E. T. Whittaker and G. Watson, $A$ course of modern analysis, Cambridge University Press, 1952.

10. A. Zygmund, A remark on characteristic functions, Proceedings of the Second Berkeley Symposium, Berkeley, University of California Press, 1951, pp. 369-372.

Columbia University,

NEW YoRK, NEW YoRK 